\title{
A limit on nuclear activity in Leo A
}

\author{
E. J. A. Meurs ${ }^{\star}$ \\ Dunsink Observatory, Castleknock, Dublin 15, Ireland \\ Received 29 March 2002 / Accepted 20 May 2003

\begin{abstract}
ROSAT HRI observations of Leo A are analysed in order to complement a recent X-ray survey of the centres of Local Group galaxies (Zang \& Meurs 2001). It is likely that high-energy cores in galaxies are indicative of some level of nuclear activity. The upper limit $0.1-2.4 \mathrm{keV}$ luminosity determined for Leo A ( $\log L_{\mathrm{X}}<35.77$ ) is consistent with the results that were obtained for other small Local Group systems. Leo A represents the lowest optical luminosity Irregular galaxy in the Local Group that has been studied at X-rays. Also the ROSAT All Sky Survey data allow to derive a (less constraining) upper limit flux for this galaxy.
\end{abstract}

Key words. galaxies: nuclei - galaxies: individual: Leo A - galaxies: Local Group - X-rays: galaxies - X-rays: individuals: Leo A

\section{Introduction}

Activity in the nuclei of galaxies is a well-established phenomenon, with its most dramatic manifestation in Quasi Stellar Objects, quasars and blazars (see e.g. Robson 1996 for an overview). Less conspicuous but more commonly found weak forms of nuclear activity can be recognized among many of the nearby galaxies. Recent surveys carried out at X-rays, covering stellar systems from our own Local Group out to $V_{\text {rad }} \lesssim$ $1000 \mathrm{~km} \mathrm{~s}^{-1}$, find evidence of energetic core sources in typically $40 \%$ of the objects investigated (Zang \& Meurs 2001; Colbert \& Mushotzky 1999; Lira et al. 2000). When specifically nearby galaxies with recognised indications for nuclear activity are considered, the detection rate increases to 60-70\% (Roberts \& Warwick 2000; Ho et al. 2001). These results are relevant for several problems concerning the general occurrence of nuclear activity in galaxies, the range of energy output of nuclear sources and the type or size of galaxies that may accommodate an active core.

The Local Group presents a target sample of particular interest, since the small distances allow low detection levels to be reached, along with comparatively high spatial resolution. The latter aspect is important in view of conceivable source confusion if activity levels comparable to Supernova Remnants (SNRs) or X-ray binaries would occur. The Local Group membership includes galaxies down to the faintest luminosity levels known. Only some of the nearest groups of galaxies offer similar opportunities (with probably even greater completeness in their coverage of small systems, see Trentham 1998). For these reasons, Zang \& Meurs (2001, hereafter ZM) recently examined the Local Group galaxies for which pointed ROSAT X-ray data are available. High-energy data like these

\footnotetext{
^ e-mail: ejam@dunsink.dias.ie
}

are generally efficient for detecting signs of central activity in galaxies, as argued in the surveys cited above. ZM covered 2/3 of the Local Group galaxies listed by Whiting et al. (1997).

The precise contents of lists of Local Group members vary however from author to author. For example, the dIrr galaxy NGC 3109 is often considered a Local Group member (Grebel 1999), but the recent extensive review by van den Bergh (1999) treats this object (along with a few other small galaxies) as members of the distinct Antlia-Sextans group. A galaxy that is not included by Whiting et al. (1997), but is nowadays confirmed as a Local Group member, is the IrV object Leo A (Tolstoy et al. 1998). Only a few other galaxies left out by Whiting et al. are possibly additional Local Group members, but only for Leo A are pointed ROSAT observations available. For the sake of greater completeness of the high-energy examination of Local Group cores, it is of interest to present an analysis of these X-ray data (with main emphasis on the central region of Leo A) and to compare the results with those obtained by ZM for the other Local Group members.

\section{X-ray data}

Leo A was observed with the ROSAT High Resolution Imager (HRI) on two occasions, in May 1992 (19.1 ks) and May $1993(11.7 \mathrm{ks})$. The HRI features better spatial resolution than the other detector onboard, the PSPC (Position Sensitive Proportional Counter): typically 6" and 22", respectively. Unlike the PSPC, the HRI has however almost no spectral resolution.

The two Leo A HRI observations were obtained from the ROSAT public archive at the Max Planck Institut für extraterrestrische Physik in Garching, Germany (respectively with frame identifiers, or ROR numbers, $600117 \mathrm{~h}$ 
Table 1. X-ray sources detected in image $600117 \mathrm{~h}$.

\begin{tabular}{|c|c|c|c|c|c|c|}
\hline$\overline{\mathrm{RA}(2000)}$ & $\overline{~ D e c ~(2000) ~}$ & $\overline{\text { Counts }( \pm)}$ & $\overline{\overline{M L}}$ & $\begin{array}{l}\text { ctrate }( \pm) \\
\left(\mathrm{s}^{-1}\right)\end{array}$ & $\begin{array}{r}\begin{array}{r}\text { offaxis } \\
(')\end{array} \\
\end{array}$ & $\begin{array}{l}\text { source } \mathrm{nr} \\
\text { in Fig. } 1\end{array}$ \\
\hline $095839.63\left( \pm 2^{\prime \prime} .2\right)$ & $+305057.6\left( \pm 2{ }^{\prime \prime} 6\right)$ & $30.3(7.5)$ & 15.2 & $0.00170(0.00042)$ & 11.2 & 7 \\
\hline $095844.03\left( \pm 33^{\prime \prime} 4\right)$ & $+305459.6\left( \pm 3^{\prime \prime} .4\right)$ & $29.7(8.4)$ & 9.8 & $0.00170(0.00048)$ & 13.2 & 4 \\
\hline $095852.84( \pm 2 \prime .5)$ & $+303624.8\left( \pm 22^{\prime \prime} 7\right)$ & $23.6(6.8)$ & 10.4 & $0.00132(0.00038)$ & 10.9 & 12 \\
\hline $095855.94\left( \pm 1^{\prime \prime} .5\right)$ & $+304941.5\left( \pm 1^{\prime \prime} .7\right)$ & $15.3(5.0)$ & 10.4 & $0.00084(0.00027)$ & 7.6 & 8 \\
\hline $095856.34\left( \pm 1^{\prime \prime} .4\right)$ & $+305237.1\left( \pm 1^{\prime \prime} .4\right)$ & $36.6(7.5)$ & 29.1 & $0.00203(0.00041)$ & 9.7 & 6 \\
\hline $095859.04( \pm 2 \prime 23)$ & $+305904.8( \pm 2 . \prime 3)$ & $91.1(12.9)$ & 48.7 & $0.00533(0.00075)$ & 15.1 & 1 \\
\hline $095859.32\left( \pm 1^{\prime \prime} .1\right)$ & $+304210.1\left( \pm 1^{\prime \prime} .1\right)$ & $15.4(4.5)$ & 16.0 & $0.00083(0.00024)$ & 6.0 & 11 \\
\hline $095941.65( \pm 2 \prime 2)$ & $+303346.5\left( \pm 2{ }^{\prime \prime} 3\right)$ & $29.9(7.6)$ & 14.6 & $0.00168(0.00043)$ & 11.9 & 13 \\
\hline $095945.33\left( \pm 1^{\prime \prime} .7\right)$ & $+304642.3\left( \pm 1^{\prime \prime} .5\right)$ & $11.2(4.0)$ & 9.1 & $0.00060(0.00022)$ & 4.9 & 10 \\
\hline $095944.29( \pm 0.8)$ & $+305622.8\left( \pm 0 .{ }^{\prime} 8\right)$ & $192.8(15.5)$ & 246.3 & $0.01093(0.00088)$ & 12.2 & 3 \\
\hline $095953.84\left( \pm 22^{\prime \prime} 5\right)$ & $+305312.6( \pm 2.3)$ & $27.2(7.2)$ & 12.9 & $0.00152(0.00040)$ & 10.4 & 5 \\
\hline $095957.40\left( \pm 3^{\prime \prime} .8\right)$ & $+305658.6\left( \pm 4^{\prime \prime} .3\right)$ & $35.7(9.6)$ & 10.6 & $0.00206(0.00055)$ & 14.0 & 2 \\
\hline $100010.95\left( \pm 1^{\prime \prime} .2\right)$ & $+304718.7\left( \pm 1^{\prime \prime} .1\right)$ & $61.0(9.0)$ & 61.1 & $0.00339(0.00050)$ & 10.4 & 9 \\
\hline
\end{tabular}

Table 2. X-ray sources detected in image $600117 \mathrm{~h} 1$.

\begin{tabular}{|c|c|c|c|c|c|c|c|}
\hline$\overline{\mathrm{RA}(2000)}$ & $\overline{\text { Dec }(2000)}$ & $\overline{\text { Counts }( \pm)}$ & $\overline{\overline{M L}}$ & $\begin{array}{l}\text { ctrate }( \pm) \\
\left(\mathrm{s}^{-1}\right)\end{array}$ & $\begin{array}{r}\text { offaxis } \\
(') \\
\end{array}$ & $\begin{array}{l}\text { source } \mathrm{nr} \\
\text { in Fig. } 2\end{array}$ & $\begin{array}{l}\text { source nr } \\
\text { in Fig. } 1\end{array}$ \\
\hline $095858.85( \pm 2.8)$ & $+305856.5( \pm 2.7)$ & $60.3(10.1)$ & 37.7 & $0.00576(0.00096)$ & 15.0 & 1 & 1 \\
\hline $095859.29\left( \pm 1^{\prime \prime} .4\right)$ & $+304205.1\left( \pm 1^{\prime \prime} .4\right)$ & $9.8(3.5)$ & 11.2 & $0.00086(0.00031)$ & 6.1 & 5 & 11 \\
\hline $095944.21( \pm 0.99)$ & $+305619.1\left( \pm 1^{\prime \prime} .0\right)$ & $142.1(13.0)$ & 218.8 & $0.01312(0.00120)$ & 12.1 & 2 & 3 \\
\hline $095953.76\left( \pm 1^{\prime \prime} .9\right)$ & $+305308.1\left( \pm 1^{\prime \prime} .8\right)$ & $23.9(5.9)$ & 19.4 & $0.00216(0.00053)$ & 10.3 & 3 & 5 \\
\hline $095959.93\left( \pm 2^{\prime \prime} .5\right)$ & $+303508.0\left( \pm 3{ }^{\prime \prime} 3\right)$ & $19.9(6.0)$ & 11.3 & $0.00183(0.00055)$ & 12.5 & 6 & - \\
\hline $100010.85\left( \pm 1^{\prime \prime} .2\right)$ & $+304713.1\left( \pm 1^{\prime \prime} .4\right)$ & $45.2(7.5)$ & 57.9 & $0.00408(0.00068)$ & 10.3 & 4 & 9 \\
\hline
\end{tabular}

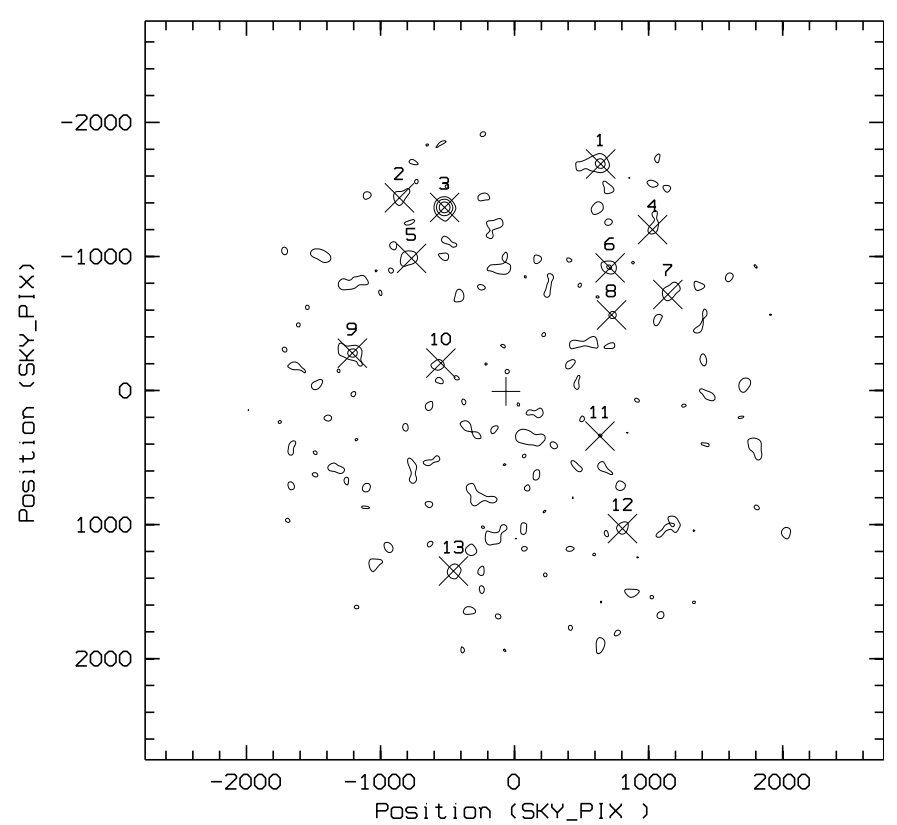

Fig. 1. Leo A ROSAT HRI frame $600117 \mathrm{~h}(18.9 \mathrm{ks})$. One sky pixel equals 0.'5. Crosses indicate sources detected down to $M L=9$. The plus sign in the middle refers to the optical position of the galaxy as listed in NED and SIMBAD. Image smoothed with 3 image pixel size Gaussians; contour levels $0.78,1.15,1.88 \mathrm{ct} \mathrm{pix}^{-1}$. Source numbers as listed in Table 1.

and 600117h1). The dates over which these observations were carried out are, respectively, 22-24 May 1992 and 1417 May 1993. The galaxy is positioned at the centre of the HRI frames. The data reduction was performed with the EXSAS/MIDAS software package (Zimmermann et al. $1998)^{1}$, according to standard procedures. The HRI images are binned at 5 ", corresponding to their typical resolution, and are corrected for vignetting and dead time. The Maximum Likelihood $(M L)$ source detection accepts sources till a minimum $M L$ of existence that constitutes a trade-off between aiming at completeness and introducing spurious detections. The $M L$ of existence is defined as $-\ln (1-P)$, where $P$ is the probability of existence of the source (Cruddace et al. 1988; Zimmermann et al. 1998). A minimum $M L$ value of 9 is applied in the present analyses, which corresponds to about 3.8 Gaussian $\sigma$. Tables 1 and 2 list the sources detected in frames $600117 \mathrm{~h}$ and $600117 \mathrm{~h} 1$, respectively. No sources with $M L>9$ were found within 4.'5 from the nominal Leo A centre (Cotton et al. 1999, as listed in NED and SIMBAD: $09^{\mathrm{h}} 59^{\mathrm{m}} 26.5$, $+30^{\circ} 44^{\prime} 47^{\prime \prime}$; epoch 2000 , estimated error 3.' 5 in each coordinate) in either frame. Therefore, an upper limit was determined in each frame at the Leo A position, using the EXSAS Upper Limit routine (which refers to a $95.4 \%$ confidence level). The results of this are in Table 3, together with the nett exposure times. There are some differences in background level between the two observations that produce a slightly higher rms noise for the frame with the longer exposure time.

Contour plots of the two HRI observations are shown in Fig. 1 (600117h, 18.9 ks) and Fig. 2 (600117h1, 11.6 ks), with source numbers as in Tables 1 and 2 . The source nearest the centre of Leo A, No. 10 in frame 600117h (Table 1),

\footnotetext{
1 http://wave.xray.mpe.mpg.de/exsas/usersguide
} 


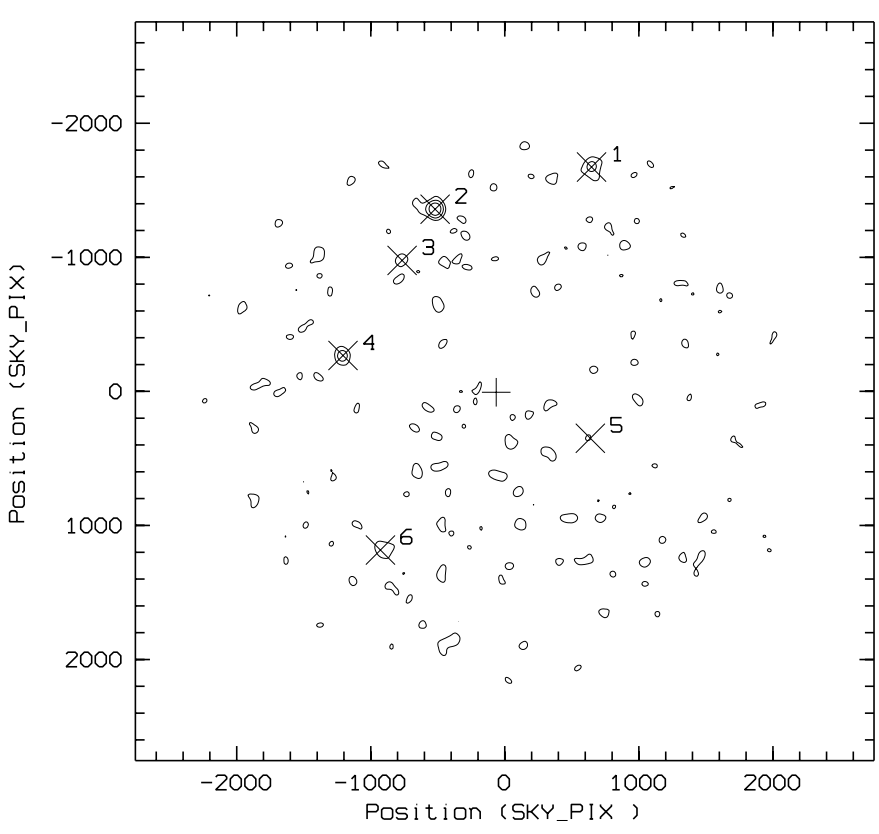

Fig. 2. Same as Fig. 1, but for HRI frame $600117 \mathrm{~h} 1$ (11.6 ks); contour levels $0.44,0.67,1.12 \mathrm{ct} \mathrm{pix}^{-1}$. Source numbers as listed in Table 2.

is indicated in Fig. 3 on a red 2nd generation POSS image of the immediate surroundings of LeoA; there is no obvious optical counterpart to this source. The big plus sign indicates the catalogued optical position of Cotton et al. (1999), the ellipse in Fig. 3 represents the 25th magnitude isophotal size from the Third Reference Catalogue of Bright Galaxies (de Vaucouleurs et al. 1991). None of the entries or catalogues included in SIMBAD and NED provide a position angle for the elliptical isophotal shape. The POSS2 image clearly shows Leo A to be elongated in roughly the East-West direction and correspondingly the position angle is taken as $90^{\circ}$ in Fig. 3 . The absense of X-ray detections associated with Leo A in the two HRI exposures is evident in all the figures. (Also if the acceptance level were lowered to $M L=6$, or 3 Gaussian $\sigma$, no sources are found within the body of Leo A.)

\section{Discussion}

The upper limits determined for Leo A in these two ROSAT HRI frames are very comparable (see Table 3); in the following the upper limit is taken as $0.0002 \mathrm{cts}^{-1}$, in order to stay on the safe side. The upper limit to the $0.1-$ $2.4 \mathrm{keV}$ unabsorbed flux is subsequently calculated under the same assumptions as for the other Local Group galaxies examined by ZM, viz. a power-law spectrum with photon index $\Gamma=2.0$ and Galactic absorption $\left(N_{\mathrm{H}}=0.179 \times 10^{21} \mathrm{~cm}^{-2}\right.$ for Leo A; Dickey \& Lockman 1990). The result, $f_{\mathrm{X}}=1.03 \times$ $10^{-14} \mathrm{erg} \mathrm{s}^{-1} \mathrm{~cm}^{-2}$, and the distance of $690 \mathrm{kpc}$ to Leo A (Tolstoy et al. 1998) lead then to an upper limit to the 0.1$2.4 \mathrm{keV}$ luminosity of a core X-ray source in Leo A of $L_{\mathrm{X}}<$ $0.58 \times 10^{36} \mathrm{erg} \mathrm{s}^{-1}$, or $\log L_{\mathrm{X}}<35.77$.

ZM (their Fig. 4) found a distribution of upper limits for 18 Local Group galaxy cores, stretching from $\log L_{X}=33.85$ to $\log L_{\mathrm{X}}=37.32$. The upper limit determined here for Leo A takes an intermediate position in this distribution, close to the
Table 3. ROSAT HRI results on Leo A centre.

\begin{tabular}{lcc}
\hline \hline Exposure & $\begin{array}{c}\text { Nett exposure time } \\
(\mathrm{s})\end{array}$ & $\begin{array}{c}\text { UL count rate } \\
\left(\mathrm{s}^{-1}\right)\end{array}$ \\
\hline $600117 \mathrm{~h}$ & 18894 & $<0.00021$ \\
$600117 \mathrm{~h} 1$ & 11612 & $<0.00017$ \\
\hline
\end{tabular}

actual median of the upper limit luminosities. Any core source in Leo A is at least a factor of 5 lower in luminosity than Sgr A* in the centre of our Galaxy, which exhibits the lowest nuclear activity level known. This comparison is based in a consequent manner on ROSAT data. Baganoff et al. (2001) have observed flaring from Sgr A* with the Chandra satellite that increases above a quiescent level by factors 20-100. The luminosities measured by Chandra are however very low, $2.2 \times 10^{33} \mathrm{erg} \mathrm{s}^{-1}$ in quiescence and up to $1.0 \times 10^{35} \mathrm{erg} \mathrm{s}^{-1}$ at peaks; flaring takes place probably less than 10 percent of the time. Using the spectral parameters derived by Baganoff et al., the luminosities in quiescence and at peak in the ROSAT band of 0.1-2.4 keV are found to be $\sim 10^{34} \mathrm{erg} \mathrm{s}^{-1}$ and up to $\sim 8 \times 10^{34} \mathrm{erg} \mathrm{s}^{-1}$, respectively. These values are smaller than the luminosity derived from the ROSAT data of Predehl \& Trümper (1994), $3.34 \times 10^{36} \mathrm{erg} \mathrm{s}^{-1}$ (ZM, Table 2), which is probably due to the greater spatial resolution of Chandra (see also Predehl et al. 1998).

It is noticed in passing that for one of the four detections considered by ZM, viz. M 31, Chandra results now have been published (Garcia et al. 2000) that might be expected to modify their results. The M 31 nuclear source that had been evident in a ROSAT HRI exposure (Primini et al. 1993) is resolved in 4 or possibly 5 separate components in the Chandra data. Using this observation from the Chandra archive $^{2}$, the proper M 31 nuclear source as identified by Garcia et al. accounts for $41 \%$ of the total flux from this complex (or slightly more, $47 \%$, if the SW-most Chandra source in this complex is a transient, as comparison between the ROSAT HRI and Chandra images suggest). Garcia et al. found however the nuclear core to have an abnormally soft X-ray spectrum. With spectral parameters as they derive for this source, for respectively a power-law or a thermal bremsstrahlung spectrum, and mean values for the other central sources, the nuclear contribution increases from $41 \%$ to $99 \%$ or $66 \%$ in the ROSAT $0.1-2.4 \mathrm{keV}$ band. These latter values for the ROSAT comparisons (but actually also the $41 \%$ ) do not alter the histogram of $\log L_{X}$ for the detections in ZM (their Fig. 4).

The absolute $V$ and $B$ magnitudes of Leo A are $M_{V}=-11.4$ and $M_{B}=-11.3$ (Mateo 1998). It has the distinction to add the lowest optical luminosity case to the Local Group Irregulars examined by ZM (see their Fig. 5). Expressed in blue solar luminosities, the value of $\log L_{B} / L_{B, \odot}=6.72$ for this galaxy without a central X-ray source is consistent with the conclusion of ZM that a minimal luminosity of around $10^{8} L_{B, \odot}$ is needed for a galaxy in order to host a core with some level of (X-ray) activity (see also Fig. 6 in ZM). Nevertheless, it may be noted that the current upper limit to the central X-ray

\footnotetext{
${ }^{2}$ http://cxc.harvard.edu/cda/
} 


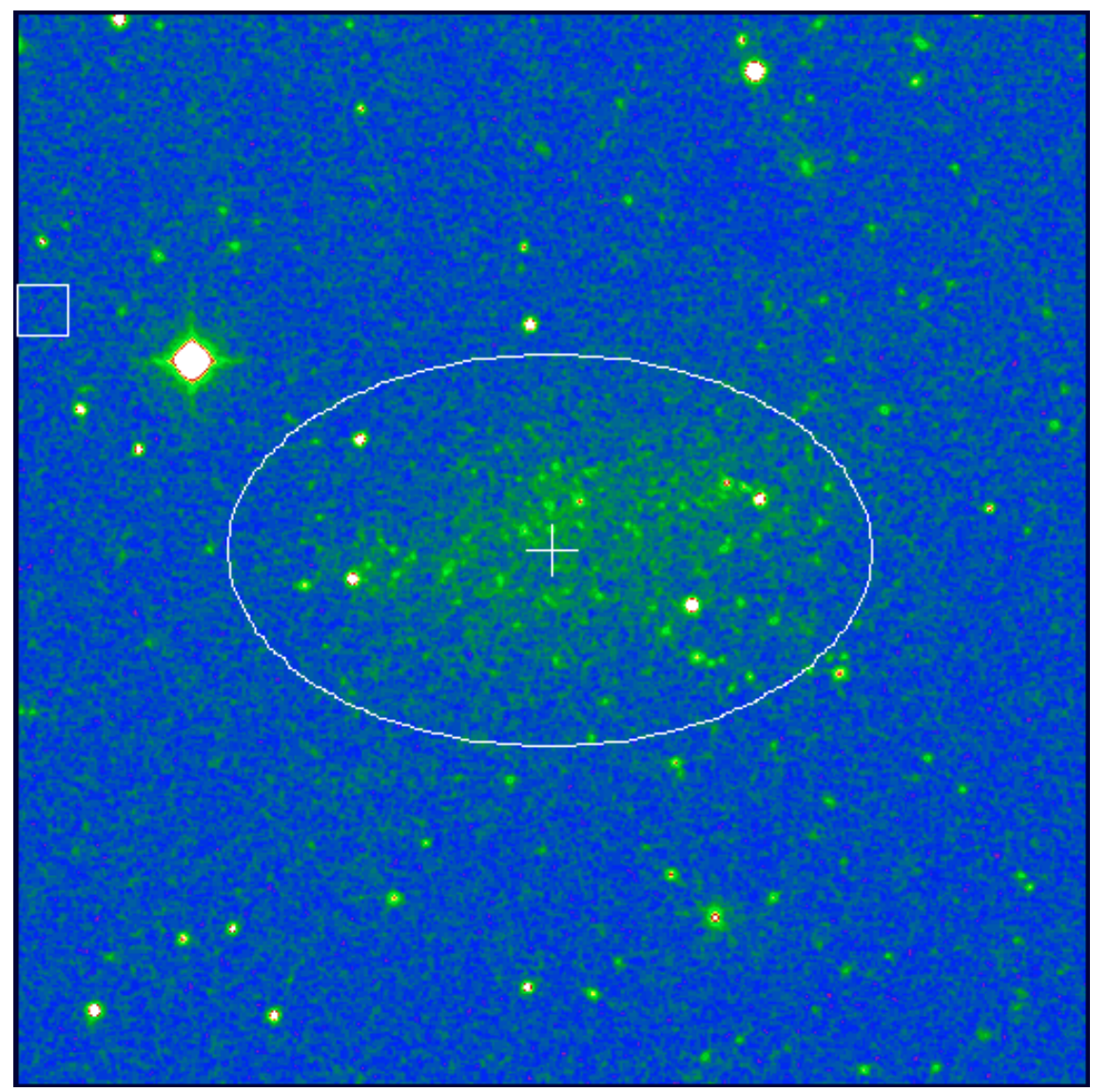

Fig. 3. POSS2 field (red) around Leo A, measuring 8.53 on a side. The plus sign indicates the optical position, the ellipse the optical size of the galaxy. The nearest source, No. 10 in the $18.9 \mathrm{ks}$ HRI exposure, is indicated with a square.

luminosity of Leo $\mathrm{A}$ is not that far below the lowest luminosity core known (Sgr $\mathrm{A}^{*}$ ) and is close to the luminosity of an intriguing but largely suspicious source that $\mathrm{ZM}$ found near the catalogued optical position of the Irregular galaxy NGC 6822. Upper limits just below $10^{36} \mathrm{erg} \mathrm{s}^{-1}$ were furthermore derived for a couple of Local Group galaxies (but with $L_{B}$ a bit higher, around $10^{8} L_{B, \odot}$ ) with weak evidence for near-central emission. Most upper limits for galaxies with $L_{B}$ around $10^{6} L_{B, \odot}$ (as for Leo A) are on the other hand one or two orders of magnitude smaller than the Leo A upper limit.

Are these considerations suggestive enough to expect detection of a weak core in Leo A when somewhat lower levels of sensitivity will be reached? Any such hope has to be moderated in view of the Irregular nature of the galaxy, which is notoriously a difficult case for defining a proper nucleus; the catalogued optical position usually only reflects a subjective estimate of a general centroid for these objects. Quite possibly there is no regular nucleus as such and future detections of $\mathrm{X}$-ray sources in Leo A could be anywhere within this galaxy. For comparison, if for the overall X-ray output of Leo A an $L_{\mathrm{X}} / L_{B}$ ratio as in Eskridge \& White (1997) is assumed, a total $L_{\mathrm{X}} \approx 1.0 \times 10^{36} \mathrm{erg} \mathrm{s}^{-1}$ is expected, which is only a factor 1.7 above the current upper limit level for individual sources $\left(0.58 \times 10^{36} \mathrm{erg} \mathrm{s}^{-1}\right)$.

It is finally noted that a couple of years earlier ROSAT looked at the Leo A region during its All Sky Survey (RASS; 1990-1991), which was carried out with the PSPC detector. The RASS data have now become accessable and 390 s exposure time is available at the Leo A position. The RASS data yield images binned in $45^{\prime \prime}$ pixels. No source could be found down to $M L=6$ for this galaxy. The EXSAS Upper Limit routine was then used to determine an upper limit of $0.012 \mathrm{cts}^{-1}$. This corresponds to an upper limit flux of $1.92 \times 10^{-13} \mathrm{erg} \mathrm{cm}^{-2} \mathrm{~s}^{-1}$ for a power law spectrum with photon index $\Gamma=2.0$ and Galactic absorption (see above), or a limiting $0.1-2.4 \mathrm{keV}$ luminosity of $1.09 \times 10^{37} \mathrm{erg} \mathrm{s}^{-1}\left(\log L_{\mathrm{X}}<37.04\right)$. Thus, the RASS upper limit is almost a factor 20 higher than, and consistent with, that determined from the HRI pointed observations.

Acknowledgements. The data analysis in this paper was carried out with the EXSAS/MIDAS data reduction package from the Max-Planck-Institut für extraterrestrische Physik in Garching. This research has made use of the SIMBAD database maintained by the Centre de Données astronomiques de Strasbourg. Also used was the NASA/IPAC Extragalactic Database (NED) which is operated by the Jet Propulsion Laboratory, California Institute of Technology, under contract with the National Aeronautics and Space Administration. The referee, Dr. R. P. Roberts, is thanked for suggesting to adopt a higher source acceptance level.

\section{References}

Baganoff, F. K., Bautz, M. W., Brandt, W. N., et al. 2001, Nature, 413, 45 
van den Bergh, S. 1999, A\&AR, 9, 273

Colbert, E. J. M., \& Mushotzky, R. F. 1999, ApJ, 519, 89

Cotton, W. D., Condon, J. J., \& Arbizzani, E. 1999, ApJS, 125, 409

Cruddace, R., Hasinger, G. R., \& Schmitt, J. H. 1988, in Astronomy from Large Databases: Scientific Objectives and Methodological Approaches, ed. F. Murtagh, \& A. Heck (München: ESO), 177

Dickey, J. M., \& Lockman, F. J. 1990, ARA\&A, 28, 215

Eskridge, P. B., \& White, R. E., III. 1997, AJ, 1114, 988

Garcia, M. R., Murray, S. S., Primini, F. A., et al. 2000, ApJ, 537, L23

Grebel, E. K. 1999, in The Stellar Content of Local Group Galaxies, ed. P. Whitelock, \& R. Cannon (San Francisco: ASP), IAU Symp., 192, 17

Ho, L. C., Feigelson, E. D., Townsley, L. K., et al. 2001, ApJ, 549, L51

Lira, P., Lawrence, A., \& Johnson, R. 2000, MNRAS, 319, 17

Mateo, M. 1998, ARA\&A, 36, 435
Predehl, P., \& Trümper, J. 1994, A\&A, 290, L29

Predehl, P., Costantini, E., Hasinger, G., \& Tanaka, Y. 2003, AN, 324, 73

Primini, F. A., Forman, W., \& Jones, C. 1993, ApJ, 410, 615

Roberts, T. P., \& Warwick, R. S. 2000, MNRAS, 315, 98

Robson, I. 1996, Active Galactic Nuclei (Chichester: John Wiley and Sons)

Tolstoy, E., Gallagher, J. S., Cole, A. A., et al. 1998, AJ, 116, 1244

Trentham, N. 1998, MNRAS, 294, 193

de Vaucouleurs, G., de Vaucouleurs, A., Corwin, H. G. Jr., et al. 1991, Third Reference Catalogue of Bright Galaxies (Berlin: Springer)

Whiting, A. B., Hau, G. K. T., \& Irwin, M. J. 1997, Spectrum, No. 14,4

Zang, Z., \& Meurs, E. J. A. 2001, ApJ, 556, 24 (ZM)

Zimmermann, U., Becker, W., Belloni, T., \& Izzo, C. 1998, EXSAS User's Guide (Garching: MPE) 